\title{
The variety of commutative additively and multiplicatively idempotent semirings
}

\author{
Ivan Chajda $^{1}$ - Helmut Länger ${ }^{2}$
}

Received: 4 February 2016 / Accepted: 21 October 2017 / Published online: 17 November 2017 (C) The Author(s) 2017. This article is an open access publication

\begin{abstract}
The variety $\mathcal{Z}$ of commutative additively and multiplicatively idempotent semirings is studied. We prove that $\mathcal{Z}$ is generated by a single subdirectly irreducible three-element semiring and it has a canonical form for its terms. Hence, $\mathcal{Z}$ is locally finite despite the fact that it is residually large. The word problem in $\mathcal{Z}$ is solvable.
\end{abstract}

Keywords Semiring · Commutative - Additively idempotent · Multiplicatively idempotent $\cdot$ Variety $\cdot$ Locally finite $\cdot$ Residually large $\cdot$ Word problem

The concept of semiring is surprisingly successful in applications both in mathematics and computer science as it was shown by Golan in [6] and Kuich and Salomaa in [8]. In this paper we concentrate on the variety $\mathcal{C}$ of commutative multiplicatively idempotent semirings which was investigated by the authors already in [2-4] and in the subvariety of this variety satisfying $x+x \approx x$. The concept of a Boolean semiring was introduced by Guzmán in [7]. It is a commutative multiplicatively idempotent semiring satisfying

Communicated by Mikhail Volkov.

Support of the research by the Austrian Science Fund (FWF), Project I 1923-N25, and the Czech Science Foundation (GAČR), Project 15-34697L, and by ÖAD, Project CZ 04/2017, as well as support of the first author by IGA, Project PřF 2017012, is gratefully acknowledged.

Helmut Länger

helmut.laenger@tuwien.ac.at

Ivan Chajda

ivan.chajda@upol.cz

1 Department of Algebra and Geometry, Faculty of Science, Palacký University Olomouc, 17. listopadu 12, 77146 Olomouc, Czech Republic

2 Faculty of Mathematics and Geoinformation, Institute of Discrete Mathematics and Geometry, TU Wien, Wiedner Hauptstraße 8-10, 1040 Vienna, Austria 
the identity $1+x+x \approx 1$. The variety $\mathcal{B}$ of Boolean semirings contains just two subdirectly irreducible members, namely the two-element (distributive) lattice and the two-element Boolean ring. On the contrary, $\mathcal{C}$ has a proper class of subdirectly irreducible members, i. e. it is residually large, as it was proved by the authors in [2]. The natural question arises if by adding another elementary identity to the identities defining $\mathcal{C}$ the number of subdirectly irreducible members can be reduced. In our previous paper we studied members of $\mathcal{C}$ satisfying $x+y+x y z \approx x+y$. In the present paper we investigate the subvariety $\mathcal{Z}$ of $\mathcal{C}$ determined by $x+x \approx x$ and make frequent use of the identity $x+y+x y \approx x+y$. That this identity indeed holds in $\mathcal{Z}$ can be seen as follows:

$$
x+y+x y \approx x^{2}+x y+y x+y^{2} \approx(x+y)^{2} \approx x+y
$$

We show that $\mathcal{Z}$ is locally finite and we provide an explicit upper bound for the cardinalities of finite free algebras in $\mathcal{Z}$. Our main result is to prove that $\mathcal{Z}$ is generated by a single three-element subdirectly irreducible semiring. Moreover, it turns out that there are single semirings of arbitrary cardinality generating $\mathcal{Z}$.

For the reader's convenience, all the concepts concerning semirings are included. What concerns concepts from Universal Algebra necessary for understanding the paper, the reader is referred to [1].

We start with the definition of a semiring in the sense of the monograph [6] by Golan.

Definition 1 A semiring is an algebra $\mathbf{S}=(S,+, \cdot, 0,1)$ of type $(2,2,0,0)$ such that

- $(S,+, 0)$ is a commutative monoid.

- $(S, \cdot, 1)$ is a monoid.

- The operation $\cdot$ is distributive with respect to + .

- $x 0=0 x=0$ for all $x \in S$

$\mathbf{S}$ is called commutative if - is commutative, additively idempotent if + is idempotent and multiplicatively idempotent if - is idempotent. Let $\mathcal{Z}$ denote the variety of commutative additively and multiplicatively idempotent semirings.

The corresponding semiring of type $(2,2)$ has been called a --distributive bisemilattice by Romanowska and a bisemilattice by Pastijn and Zhao. McKenzie and Romanowska [9] showed that the variety of all bisemilattices has exactly 5 subvarieties. Based on this result, Ghosh et al. [5] and Pastijn [10] have shown that the variety of all semirings with type $(2,2)$ whose additive semigroup is a semilattice and multiplicative semigroup is a band has exactly 78 subvarieties. In this process, the identity $x+y+x y \approx x+y$ plays a role.

Every bounded distributive lattice $\mathbf{L}=(L, \vee, \wedge, 0,1)$ is a semiring in $\mathcal{Z}$. On the contrary, non-trivial Boolean rings with unit 1 do not belong to $\mathcal{Z}$ since in every nontrivial Boolean ring with 1 we have $1+1+1 \cdot 1=1 \neq 0=1+1$. In the following we present an example of a semiring in $\mathcal{Z}$ which is not a lattice since it does not satisfy the absorption law. 
Example 2 The algebra $\mathbf{S}_{3}:=\left(S_{3},+, \cdot, 0,1\right)$ with $S_{3}=\{0, a, 1\}$,

\begin{tabular}{|c|c|c|c|c|c|c|c|c|}
\hline+ & 0 & $a$ & 1 & & • & 0 & $a$ & 1 \\
\hline 0 & 0 & $a$ & $\overline{1}$ & and & $\overline{0}$ & 0 & 0 & 0 \\
\hline$a$ & $a$ & $a$ & $a$ & aाn & $a$ & 0 & $a$ & $a$ \\
\hline 1 & 1 & $a$ & 1 & & 1 & 0 & $a$ & 1 \\
\hline
\end{tabular}

belongs to $\mathcal{Z}$. Because of $1(1+a)=a \neq 1, \mathbf{S}_{3}$ is not a lattice. Let us remark that $\mathbf{S}_{3}$ satisfies the so-called weak-absorption law $x(x+y) \approx x+x y$.

It is easy to see that $\mathbf{S}_{3}$ is the unique semiring in $\mathcal{Z}$ with cardinality less than 4 which is not a lattice.

Now we repeat the definitions of certain members of $\mathcal{Z}$ introduced in [2]:

Definition 3 For every integer $n>1$ let $\mathbf{S}_{n}$ be the semiring with universe $S_{n}=$ $\{1, \ldots, n\}$ and operations defined as follows:

$$
x+y:= \begin{cases}\max (x, y) & \text { for } x \text { and } y \text { are odd } \\ y & \text { for } x \text { is odd and } y \text { is even } \\ x & \text { for } x \text { is even and } y \text { is odd } \\ \min (x, y) & \text { for } x \text { and } y \text { are even }\end{cases}
$$

and

$$
x y:=\min (x, y)
$$

$\left(x, y \in S_{n}\right.$ ). Here the operation symbols 0 and 1 are interpreted as 1 and $n$, respectively. Moreover, for every infinite cardinal $k$ let $\mathbf{C}_{k}=\left(C_{k}, \leq, 0,1\right)$ be a bounded chain of cardinality $k$ and $\mathbf{U}_{k}$ be the semiring with universe $U_{k}:=C_{k} \times\{1,2\}$ and operations defined as follows:

$$
(x, i)+(y, j):= \begin{cases}(\max (x, y), 1) & \text { for }(i, j)=(1,1) \\ (y, 2) & \text { for }(i, j)=(1,2) \\ (x, 2) & \text { for }(i, j)=(2,1) \\ (\min (x, y), 2) & \text { for }(i, j)=(2,2)\end{cases}
$$

and

$$
(x, i)(y, j):= \begin{cases}(x, i) & \text { for } x<y \\ (x, \min (i, j)) & \text { for } x=y \\ (y, j) & \text { for } x>y\end{cases}
$$

$\left((x, i),(y, j) \in U_{k}\right)$. Here the operation symbols 0 and 1 are interpreted as $(0,1)$ and $(1,2)$, respectively.

It is easy to see that the semiring $\mathbf{S}_{3}$ of Example 2 is isomorphic to the semiring $\mathbf{S}_{3}$ of Definition 3. 
It was proved in [2] that $\mathbf{S}_{3}$ is subdirectly irreducible. Moreover, in this paper it was shown that for every integer $n>1$ the semiring $\mathbf{S}_{n}$ is a subdirectly irreducible member of $\mathcal{Z}$ of cardinality $n$ and that for every infinite cardinal $k$ the semiring $\mathbf{U}_{k}$ is a subdirectly irreducible member of $\mathcal{Z}$ of cardinality $k$. Hence $\mathcal{Z}$ is residually large.

We are going to derive a canonical form of terms in $\mathcal{Z}$.

In the following let $n$ denote an arbitrary, but fixed non-negative integer and put $N:=\{1, \ldots, n\}$.

Lemma 4 Every term $t\left(x_{1}, \ldots, x_{n}\right)$ in $\mathcal{Z}$ can be written in the form

$$
t_{A}\left(x_{1}, \ldots, x_{n}\right)=\sum_{I \in A} \prod_{s \in I} x_{s} \text { with } A \subseteq 2^{N}
$$

where the empty sum is defined as 0 and the empty product as 1 .

Proof Let $W$ denote the set of all sums of products of elements of $\left\{x_{1}, \ldots, x_{n}\right\}$. Obviously, the elements of $W$ are terms in $\mathcal{Z}$. It is easy to see that $0,1 \in W$ and that the sum of two elements of $W$ again belongs to $W$. Using the distributive law one can see that also the product of two elements of $W$ again belongs to $W$. Idempotency of addition and multiplication shows that the elements of $W$ can be written in the form (1).

Corollary 5 There exist only finitely many different terms in $\mathcal{Z}$ of fixed finite arity which means that $\mathcal{Z}$ is locally finite. The number of different $n$-ary terms in $\mathcal{Z}$ is at $\operatorname{most} 2^{2^{n}}$.

In fact, one can show that $\mathcal{Z}$ is locally finite by another method. Indeed, if $\mathbf{S}$ is a semiring whose additive semigroup is a semilattice, then $\mathbf{S}$ is locally finite if and only if $(S, \cdot)$ is locally finite. It is well-known that every semilattice is locally finite.

Within $\mathcal{Z}$ we can write terms in a more economic way.

Definition 6 A subset $A$ of $2^{N}$ is called reduced if there does not exist an integer $k>2$ and pairwise different elements $I_{1}, \ldots, I_{k}$ of $A$ with $I_{1} \cup \cdots \cup I_{k-1}=I_{k}$. Let $\mathbb{A}$ denote the set of all reduced subsets of $2^{N}$. The term $t_{A}$ is called reduced if $A \in \mathbb{A}$.

At first, we show that the identity $x+y+x y \approx x+y$ can be extended to arbitrary products of variables as follows:

Lemma 7 We have

$$
\mathcal{Z} \models \prod_{i \in I} x_{i}+\prod_{i \in J} x_{i} \approx \prod_{i \in I} x_{i}+\prod_{i \in J} x_{i}+\prod_{i \in I \cup J} x_{i}
$$

for all $I, J \subseteq N$.

Proof

$$
\mathcal{Z} \models \prod_{i \in I} x_{i}+\prod_{i \in J} x_{i} \approx \prod_{i \in I} x_{i}+\prod_{i \in J} x_{i}+\prod_{i \in I} x_{i} \prod_{i \in J} x_{i} \approx \prod_{i \in I} x_{i}+\prod_{i \in J} x_{i}+\prod_{i \in I \cup J} x_{i}
$$


In the following we are going to show that every term in $\mathcal{Z}$ can be reduced.

Theorem 8 To every $A \subseteq 2^{N}$ there exists some $B \in \mathbb{A}$ with $\mathcal{Z} \models t_{A} \approx t_{B}$.

Proof Let $A \subseteq 2^{N}$. If $A \in \mathbb{A}$ then we are done. Hence assume $A \notin \mathbb{A}$. Then there exist an integer $k>2$ and pairwise different elements $I_{1}, \ldots, I_{k}$ of $A$ with $I_{1} \cup \cdots \cup I_{k-1}=$ $I_{k}$. Then by Lemma 7 we have

$$
\begin{aligned}
\mathcal{Z} & =\prod_{i \in I_{1}} x_{i}+\cdots+\prod_{i \in I_{k}} x_{i} \approx \prod_{i \in I_{1}} x_{i}+\prod_{i \in I_{2}} x_{i}+\prod_{i \in I_{1} \cup I_{2}} x_{i}+\prod_{i \in I_{3}} x_{i}+\cdots+\prod_{i \in I_{k}} x_{i} \approx \\
& \approx \prod_{i \in I_{1}} x_{i}+\prod_{i \in I_{2}} x_{i}+\prod_{i \in I_{1} \cup I_{2}} x_{i}+\prod_{i \in I_{3}} x_{i}+\prod_{i \in I_{1} \cup I_{2} \cup I_{3}} x_{i}+\prod_{i \in I_{4}} x_{i}+\cdots+\prod_{i \in I_{k}} x_{i} \approx \ldots \approx \\
& \approx \prod_{i \in I_{1}} x_{i}+\prod_{i \in I_{2}} x_{i}+\prod_{i \in I_{1} \cup I_{2}} x_{i}+\prod_{i \in I_{3}} x_{i}+\cdots+\prod_{i} x_{i-2}+\prod_{i \in I_{k-1}} x_{i} \approx \\
& \approx \prod_{i \in I_{1}} x_{i}+\prod_{i \in I_{2}} x_{i}+\prod_{i \in I_{1} \cup I_{2}} x_{i}+\prod_{i \in I_{3}} x_{i}+\cdots+\prod_{i \in I_{1} \cup \cdots \cup I_{k-2}} x_{i}+\prod_{i \in I_{k-1}} x_{i} \approx \ldots \approx \\
& \approx \prod_{i \in I_{1}} x_{i}+\prod_{i \in I_{2}} x_{i}+\prod_{i \in I_{1} \cup I_{2}} x_{i}+\prod_{i \in I_{3}} x_{i}+\prod_{i \in I_{1} \cup I_{2} \cup I_{3}} x_{i}+\prod_{i \in I_{4}} x_{i}+\cdots+\prod_{i \in I_{k-1}} x_{i} \approx \\
& \approx \prod_{i \in I_{1}} x_{i}+\prod_{i \in I_{2}} x_{i}+\prod_{i \in I_{1} \cup I_{2}} x_{i}+\prod_{i \in I_{3}} x_{i}+\cdots+\prod_{i \in I_{k-1}} x_{i} \approx \prod_{i \in I_{1}} x_{i}+\cdots+\prod_{i \in I_{k-1}} x_{i}
\end{aligned}
$$

and hence

$$
\mathcal{Z} \models t_{A} \approx t_{A \backslash\left\{I_{k}\right\}}
$$

Either $A \backslash\left\{I_{k}\right\} \in \mathbb{A}$ or again one element of $A \backslash\left\{I_{k}\right\}$ can be cancelled. After a finite number of steps one finally ends up with some $B \in \mathbb{A}$ satisfying $\mathcal{Z}=t_{A} \approx t_{B}$ (every subset of $2^{N}$ having less that three elements automatically belongs to $\mathbb{A}$ ).

Corollary 9 For $n=0,1,2$ resp. 3 there are exactly 2, 4, 14 resp. 122 reduced $n$-ary terms in $\mathcal{Z}$.

Proof The proof is evident.

Now we state and prove our main theorem saying that $\mathcal{Z}$ is generated by $\mathbf{S}_{3}$.

Theorem 10 If $A, B \in \mathbb{A}$ then $\mathbf{S}_{3} \models t_{A} \approx t_{B}$ if and only if $A=B$.

Proof Assume $t_{A} \approx t_{B}$ and $A \neq B$. Without loss of generality $A \backslash B \neq \emptyset$. Let $I \in A \backslash B$. Since $I=\emptyset$ would imply $1=t_{A}(0, \ldots, 0)=t_{B}(0, \ldots, 0)=0$ we have $I \neq \emptyset$. Let $i \in I$. Put

$$
a_{j}:= \begin{cases}0 & \text { if } j \in N \backslash I \\ a & \text { if } j=i \\ 1 & \text { if } j \in I \backslash\{i\}\end{cases}
$$


Then $t_{A}\left(a_{1}, \ldots, a_{n}\right)=a$. Hence we have $t_{B}\left(a_{1}, \ldots, a_{n}\right)=a$. From this we conclude that there exists some $J_{i} \in B$ with $i \in J_{i} \subseteq I$. Since $I \notin B$ we have $J_{i} \varsubsetneqq I$. Now define

$$
b_{j}:= \begin{cases}0 & \text { if } j \in N \backslash J_{i} \\ a & \text { if } j=i \\ 1 & \text { if } j \in J_{i} \backslash\{i\}\end{cases}
$$

Then $t_{B}\left(b_{1}, \ldots, b_{n}\right)=a$. Hence $t_{A}\left(b_{1}, \ldots, b_{n}\right)=a$ which shows that there exists some $K_{i} \in A$ with $i \in K_{i} \subseteq J_{i}$. Therefore $i \in K_{i} \varsubsetneqq I$. Obviously,

$$
I=\bigcup_{i \in I} K_{i}
$$

Let $K_{1}, \ldots, K_{s}$ denote the pairwise distinct elements of $\left\{K_{i} \mid i \in I\right\}$. Then $s>1$, $K_{1}, \ldots, K_{s}, I$ are pairwise distinct and $K_{1} \cup \cdots \cup K_{s}=I$. This contradicts $A \in \mathbb{A}$. Hence $A=B$.

Remark 11 It follows from Theorem 10 that $B$ in Theorem 8 is uniquely determined by $A$. So we could write $B=f(A)$ in Theorem 8 . For $A, B \subseteq 2^{N}$ we then have $\mathcal{Z} \models t_{A} \approx t_{B}$ if and only if $f(A)=f(B)$. Thus, the word problem in $\mathcal{Z}$ is solvable.

Remark 12 From Corollary 9 and Theorem 10 we conclude that the free algebras in $\mathcal{Z}$ with $0,1,2$ resp. 3 free generators have 2, 4, 14 resp. 122 elements.

From Theorem 10 we finally obtain

Theorem $13 \mathcal{Z}$ is generated by $\mathbf{S}_{3}$. Moreover, $\mathcal{Z}$ is generated by every $\mathbf{S}_{n}$ for each integer $n>2$ and by every $\mathbf{U}_{k}$ for each infinite cardinal $k$.

Proof Let $A, B \subseteq 2^{N}$ and assume $\mathbf{S}_{3}=t_{A} \approx t_{B}$. According to Theorem 8 there exist $C, D \in \mathbb{A}$ with $\mathcal{Z} \models t_{C} \approx t_{A}$ and $\mathcal{Z} \models t_{D} \approx t_{B}$. We conclude $\mathbf{S}_{3} \models t_{C} \approx t_{D}$. According to Theorem 10 we obtain $C=D$ and hence $\mathcal{Z} \models t_{A} \approx t_{C}=t_{D} \approx t_{B}$. Therefore $\mathbf{S}_{3}$ generates $\mathcal{Z}$. If $n>2$ is an integer then $\mathbf{S}_{n}$ has a subalgebra being isomorphic to $\mathbf{S}_{3}$, namely the subalgebra with base set $\{1,2, n\}$. Hence, every identity holding in $\mathbf{S}_{n}$ also holds in $\mathcal{Z}$. Analogously, if $k$ is an infinite cardinal then $\mathbf{U}_{k}$ has a subalgebra being isomorphic to $\mathbf{S}_{3}$, namely the subalgebra with base set $\{(0,1),(0,2),(1,2)\}$. Hence, every identity holding in $\mathbf{U}_{k}$ also holds in $\mathcal{Z}$. This completes the proof of the theorem.

Remark 14 As mentioned in Remark 12 and Corollary 9, the free algebra $\mathbf{F}_{\mathcal{Z}}(x)$ with one free generator $x$ in $\mathcal{Z}$ consists just of the four elements $0,1, x, 1+x$. It is easy to see that the equivalence relation $\Theta$ with classes $\{0\},\{x, 1+x\},\{1\}$ is a congruence on $\mathbf{F}_{\mathcal{Z}}(x)$ and $\left(\mathbf{F}_{\mathcal{Z}}(x)\right) / \Theta \cong \mathbf{S}_{3}$. This shows that also $\mathbf{F}_{\mathcal{Z}}(x)$ generates $\mathcal{Z}$.

Acknowledgements Open access funding provided by Austrian Science Fund (FWF). The authors are very grateful to an anonymous referee for valuable remarks improving the quality of the paper. 
Open Access This article is distributed under the terms of the Creative Commons Attribution 4.0 International License (http://creativecommons.org/licenses/by/4.0/), which permits unrestricted use, distribution, and reproduction in any medium, provided you give appropriate credit to the original author(s) and the source, provide a link to the Creative Commons license, and indicate if changes were made.

\section{References}

1. Bergman, C.: Universal Algebra. Fundamentals and Selected Topics. Taylor \& Francis, Boca Raton (2012)

2. Chajda, I., Länger, H.: Subdirectly irreducible commutative multiplicatively idempotent semirings. Algebra Universalis 76, 327-337 (2016)

3. Chajda, I., Länger, H.: On a variety of commutative multiplicatively idempotent semirings. Semigroup Forum 94, 610-617 (2017)

4. Chajda, I., Länger, H., Švrček, F.: Multiplicatively idempotent semirings. Math. Bohem. 140, 35-42 (2015)

5. Ghosh, S., Pastijn, F., Zhao, X.Z.: Varieties generated by ordered bands. I. Order 22, 109-128 (2005)

6. Golan, J.S.: Semirings and Their Applications. Kluwer, Dordrecht (1999)

7. Guzmán, F.: The variety of Boolean semirings. J. Pure Appl. Algebra 78, 253-270 (1992)

8. Kuich, W., Salomaa, A.: Semirings, Automata, Languages. Springer, Berlin (1986). ISBN 3-54013716-5

9. McKenzie, R., Romanowska, A.: Varieties of --distributive bisemilattices. Contr. Gen. Algebra 1, 213-218 (1979)

10. Pastijn, F.: Varieties generated by ordered bands. II. Order 22, 129-143 (2005) 